\title{
Viktor Borisovich Lidskii
}

On July 29, 2008, Viktor Borisovich Lidskii, a wonderful person and a distinguished mathematician whose first-class results enriched mathematical physics and operator theory, passed away at the age of 84 after a prolonged and painful illness.

At the end of World War II, Viktor Borisovich served as a reconnaissance officer and was decorated with many orders and medals.

Viktor Borisovich entered mathematics with remarkable achievements. By means of subtle investigations of periodic Hamiltonian systems, he proved that the well-known sufficient conditions for stability are also necessary. He was the first to start a systematic study of spectral properties of nonself-adjoint SturmLiouville operators. His theorem on the coincidence of the matrix and spectral traces for trace class operators in a Hilbert space is a classical result. His method for the summation of Fourier series over root vectors of nonself-adjoint operators, which he called the Abel method, is now called the Abel-Lidskii method.

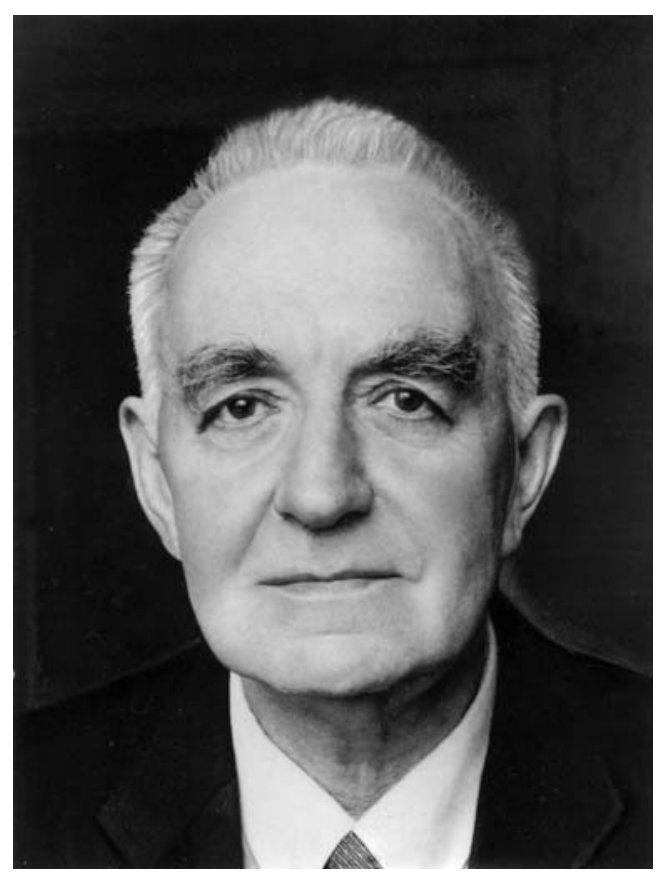

Lidskii, together with a team of collaborators, studied the natural vibration frequency spectrum of membrane shells and discovered intervals of continuous spectrum. He investigated the difficult problem on the vibrations of a closed shell in a space filled with a viscous fluid.

Viktor Borisovich was an energetic but modest and sympathetic person; his students and numerous postgraduates liked and respected him, and everybody was happy to be close to him and communicate with him informally.

He knew and loved classical art, and his interests outside mathematics were also very wide.

Viktor Borisovich Lidskii was an active member of the editorial board of our journal since its foundation almost until the end of his life. We shall always remember him gratefully.

Editorial Board 